\title{
An Overview of Dynamic Adaptive Streaming over HTTP (DASH) applications over Information-Centric Networking (ICN)
}

\author{
Koffka Khan
}

Department of Computing and Information Technology the University of the West Indies, Trinidad and Tobago, W.I Email: koffka.khan@gmail.com

Wayne Goodridge

Department of Computing and Information Technology The University of the West Indies, Trinidad and Tobago, W.I Email: wayne.goodridge@sta.uwi.edu.com

\section{ABSTRACT}

ICN has received a lot of consideration in recent years, and is a likely approach for the Future Internet design. As multimedia is the dominating traffic, it is vital to study this type of data transmission in the context of ICN. In particular, the adaptive streaming of multimedia content is a promising approach for treatment within ICN. The client has full control over the streaming session. This enables the possibility of adapting the multimedia stream to its context (e.g. network conditions, device capabilities, user-QoE (Quality of Service)). These objectives are wellmatched with the standards accepted by ICN. In this article we investigate dynamic adaptive video streaming over HTTP (DASH) over networks adopting the ICN approach. In particular, we focus on seven main applications: (1) Mobile, (2) SVC-based (Scalable Video Coding), (3) Named Data Networking (NDN), (4) Vehicular Networks, (5) 5G systems, (6) Internet of Things (IoTs), and (7) Security.

Keywords - ICN; Future Internet; DASH; Mobile; SVC; NDN; Vehicular; 5G; IoT; Security.

\section{INTRODUCTION}

Information centric networking is an emerging networking paradigm that envisages to solve the shortcomings of the host centric Internet. The usage of Internet has moved away from the initial host centric remote access one to predominantly an information serving one. In the new paradigm, information rather than the host takes the central place in serving the users [5] Hence the users will search for information and download it from the nearest device holding it. In order to serve the customers better while optimizing the use of network resources, in-network caching is enabled in ICN. In ICN, the intermediate routers will serve as cache repositories in addition to acting as data forwarding devices.
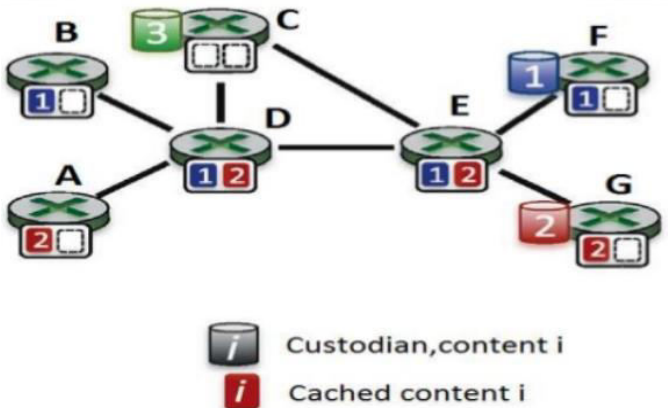

Fig. 1.Information-Centric Network (ICN) [6].

Media streaming over HTTP and, in a further consequence, streaming over the TCP, has become omnipresent in today's Internet. Content providers such as
Netflix, Hulu, and Vudu do not deploy their own streaming equipment: they use the existing Internet infrastructure as it is and simply deploy their own services Over The Top (OTT). This streaming approach works surprisingly well without any particular support from the underlying network due to the use of efficient video compression, Content Delivery Networks (CDNs), and adaptive video players. Earlier video streaming research mostly recommended use of the User Datagram Protocol (UDP) combined with the Real-time Transport Protocol (RTP). It assumed it would not be possible to transfer multimedia data smoothly with TCP, because of its throughput variations and large retransmission delays. This point of view has significantly evolved today. HTTP streaming, and especially its most simple form known as progressive download, has become very popular over the past few years because it has some major benefits compared to RTP streaming. As a consequence of the consistent use of HTTP for this streaming method, the existing Internet infrastructure consisting of proxies, caches, and CDNs could be used. Originally, this architecture was designed to support best-effort delivery of files and not real-time transport of multimedia data. Nevertheless, real-time streaming based on HTTP could also take advantage of this architecture, in comparison to RTP, which could not leverage any of the aforementioned components. Another benefit that results from the use of HTTP is that the media stream could easily pass firewalls or Network Address Translation (NAT) gateways, which was definitely a key for the success of HTTP streaming. However, HTTP streaming (see Figure 2) is not the holy grail of streaming as it also introduces some drawbacks 
compared to RTP [3]. Nevertheless, in an ICN-based video streaming architecture these aspects also have to be considered.

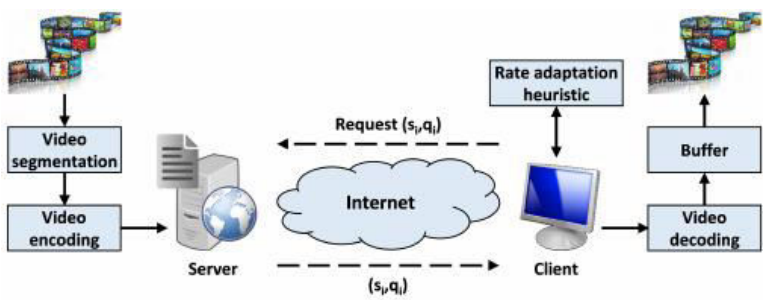

Fig. 2. Adaptive video streaming [13].

ICN and MPEG-DASH have several elements in common [8]: (1) the client-initiated pull approach; (2) the content being dealt with in pieces (or chunks); (3) the support of efficient replication and distribution of content pieces within the network; (4) the scalable, session-free nature of the exchange between the client and the server at the streaming layer: the client is free to request any chunk from any location; and (5) the support for potentially multiple source locations.

This work consists of four sections. Section II presents a categorization of DASH applications over ICN. Section III gives examples of DASH applications over ICN. Finally, the conclusion is given in Section IV.

\section{DASH APPLICATIONS OVER ICN}

DASH applications over ICN are categorized into Mobile, VC-based, Named Data Networking (NDN), Vehicular Networks, 5G Systems, Internet of Things (IoTs) and Security (see Figure 3).

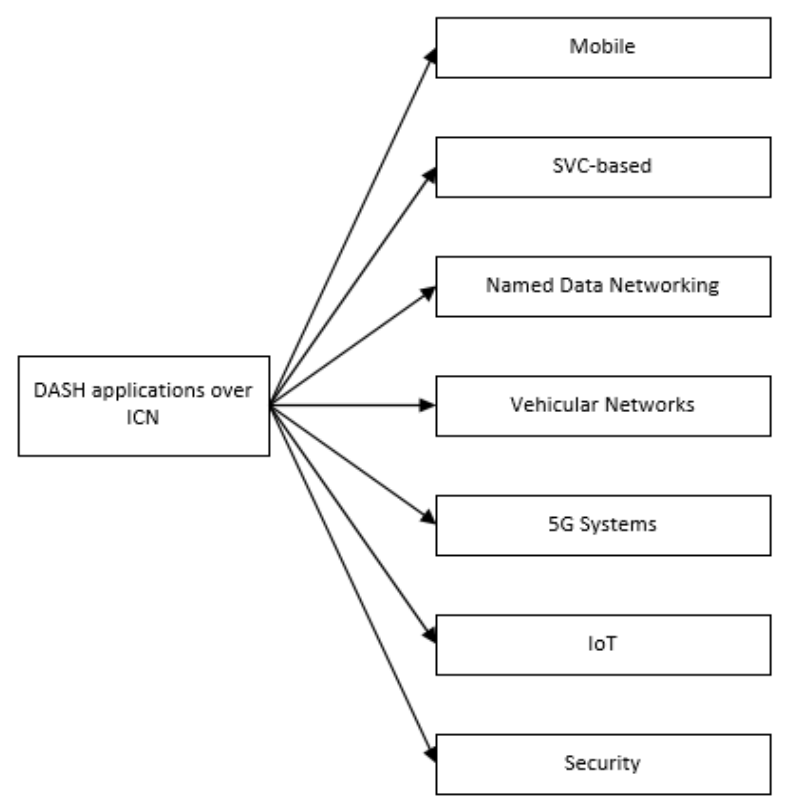

Fig. 3.DASH applications over ICN.

\section{EXAMPLES OF DASH APPLICATIONS OVER ICN}

\section{A. Mobile}

Mobile video delivery drives Internet traffic evolution and puts colossal pressure on future $5 \mathrm{G}$ networks to support higher quality and lower latency requirements over an increasingly heterogeneous network access. Future Internet paradigms recentering communication around content, such as Information Centric Networks (ICN), appear as promising candidates to relieve the challenges of a mobility-robust, efficient and cost-effective video delivery, by integrating video-awareness at network layer. Authors focus on ICN-enabled Dynamic Adaptive Streaming (DAS) over an heterogeneous wireless access. They integrate ICN capabilities in DAS clients requesting $4 \mathrm{~K}$ video content to standard DAS servers [12]. Authors deploy a virtualized ICN-enabled network slice using LXC containers to connect clients to servers through an heterogeneous wireless access (802.11n and LTE emulated radios) and a simplified backhaul (see Figure 4). The contribution of the demo is twofold. First it showcases what ICN can bring to DAS over a mobile heterogeneous access in virtue of its content-awareness at network layer. Second, it offers to the user a rich sandbox where several state-of-the-art DAS controllers are implemented and can be tested over ICN or standard TCP.

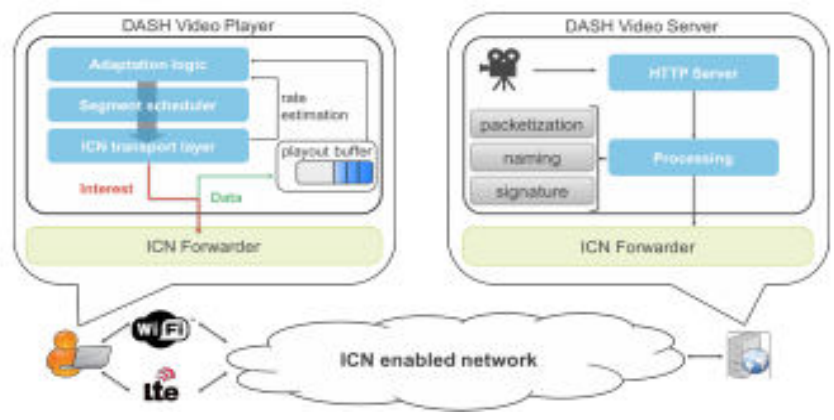

Fig. 4.ICN-enabled video architecture [12].

Information Centric Networking (ICN) is a network paradigm alternative to the classic hostcentric communication model: it provides users with content exposed as names, instead of providing communication channels between hosts. Authors present a peer-to-peer application for live streaming of video content encoded at multiple bit rates [3]. The application enables a small set of neighbouring cellular/Wi-Fi devices to increase the quality of video playback by using the Wi-Fi network to share the portion of the live stream downloaded by each peer via the cellular network (see Figure 6). The application exploits the main functionalities of ICN: routing by name, in network caching and multicast delivery. Authors include the implementation of a Java prototype of the application on a test-bed composed by Linux machines running the $\mathrm{CCNx}$ tool and streaming MPEG-DASH videos. They measure the performance of our solution and verified on the field that ICN simplifies the development of applications, as it provides built-in functions, which would be much more difficult to implement by relying on classical TCP/IP tools only. 


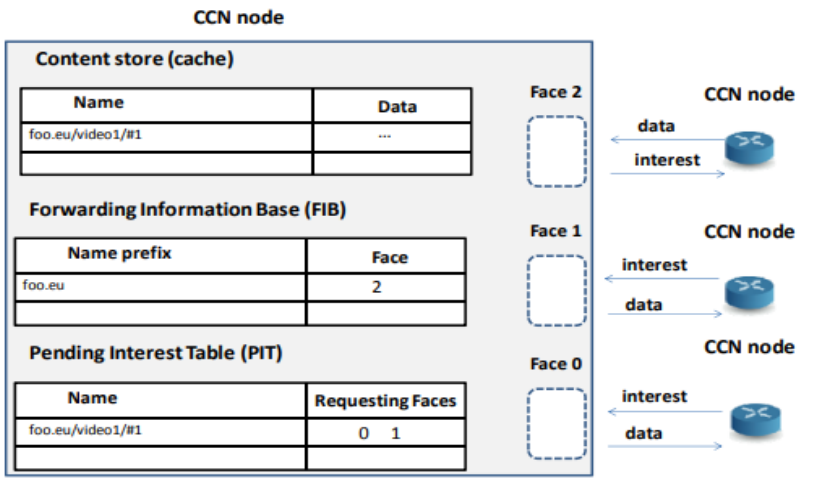

Fig. 5. CCN node [4]

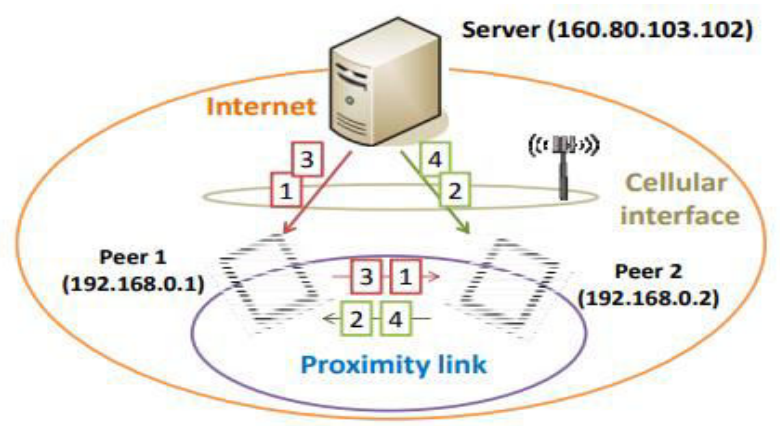

Fig. 6. Application scenario [4].

Nowadays, multimedia is omnipresent in the Internet and generates the major total traffic in fixed and mobile networks. While video streaming services become more crucial for mobile users, their traffic may often exceed the bandwidth capacity of cellular networks. Content Centric Networking $(\mathrm{CCN})$ can be an attractive solution which adapts the network architecture to the current network usage pattern. Authors propose a CCN peer-to-peer videoon-demand streaming protocol based on scalable video coding [2]. They implement a collaborative strategy which improves the video segments availability in the network and reduces latency relying on $\mathrm{CCN}$ functionalities such as caching and routing by name (see Figure 7). Authors also propose a control strategy allowing to scale to highly dynamic networks. Through the tests carried out to evaluate the performance of our solution, authors show its effectiveness. Indeed, it reduces significantly the initial playback delay and enhances the streaming quality compared to a traditional service with no collaboration policy.

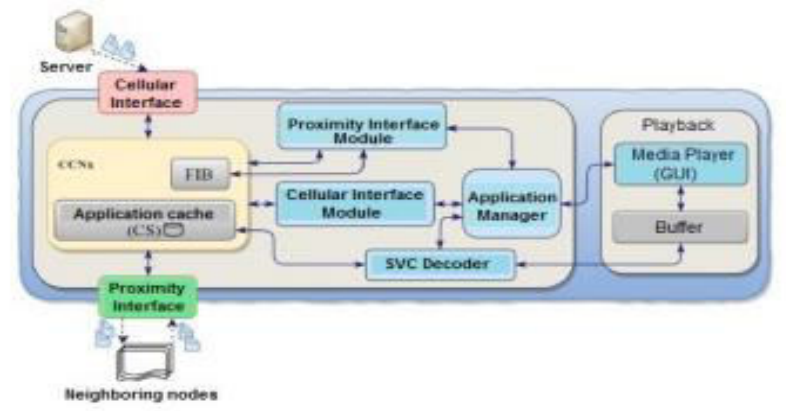

Fig. 7.Peer structure [2].
Authors present an Android application exploiting a collaborative multi-RAT approach (cellular and WiFi Radio Access Technologies) for adaptively streaming video content. The application has been developed and tested on real Android devices of different vendors. Each device coordinates with the others to download portions of a video stream through its own preferred RAT, and shares the results with the neighboring devices through a proximity, high bit rate network based on Wi-Fi Direct (see Figure 8). As a result, the aggregate bandwidth available to the group of collaborating devices allows a faster download and a higher quality of video playback streaming, with respect to what is achievable by a single device. The application is designed to exploit common Android devices along with the key functionalities of an Information Centric Network: routing-by-name, innetwork caching and multicasting.

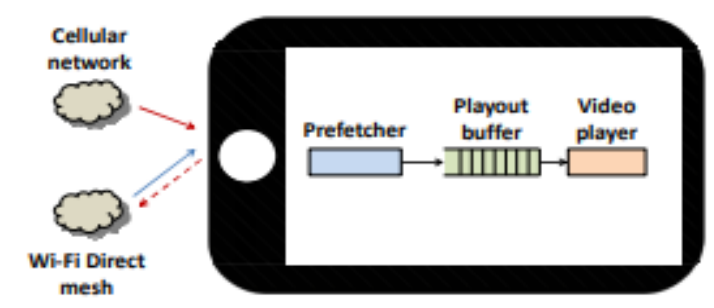

Fig. 8. The structure of a video peer [7].

\section{B. SVC-based}

HTTP Adaptive Streaming (HAS) is becoming the defacto standard for video streaming services. In HAS, each video is segmented and stored in different qualities. The client can dynamically select the most appropriate quality level to download, allowing it to adapt to varying network conditions. As the Internet was not designed to deliver such applications, optimal support for multimedia delivery is still missing. Information Centric Networking (ICN) is a recently proposed disruptive architecture that could solve this issue, where the focus is given to the content rather than to end-to-end connectivity. Due to the bandwidth unpredictability typical of ICN, standard AVC-based HAS performs quality selection sub-optimally, thus leading to a poor Quality of Experience (QoE). Authors propose to overcome this inefficiency by using Scalable Video Coding (SVC) instead (see Figures 9 and 10). They individuate the main advantages of SVC-based HAS over ICN and outline, both theoretically and via simulation, the research challenges to be addressed to optimize the delivered QoE.
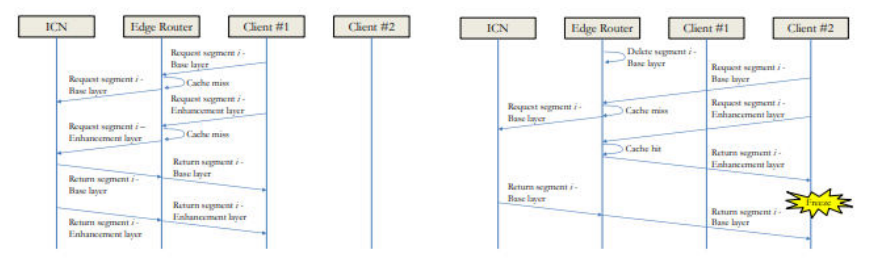

Fig. 9. An example of the negative effects caused by cache misses for the base layer of SVC-based DASH [9]. 


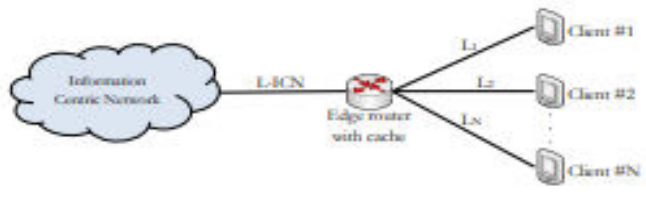

Fig. 10. Schematic representation of a communication network with $\mathrm{N}$ clients streaming video [9].

\section{C.Named Data Networking}

Named Data Networking (NDN) is a general proposed network layer protocol which offers a set of rich functionality: in-network storage, multi-path forwarding, and data-centric security. The cache and multi-path feature improves the efficiency of transmission but increases the difficulty of the bandwidth estimation. Authors present a dynamic layer switching strategy using Lyapunov Optimization is proposed for dynamic adaptive scalable video streaming over NDN (see Figure 11 and 12). It aims for optimizing long-term quality-of-experience (QoE) of users via maximizing video quality subject to a constraint on playback smoothness, interruption and fluctuation. The proposed algorithm does not require a priori knowledge of the network bandwidth dynamics and is capable of operating online with the client's currently available information. Experimental results indicate that the algorithm performs better than others in NDN.

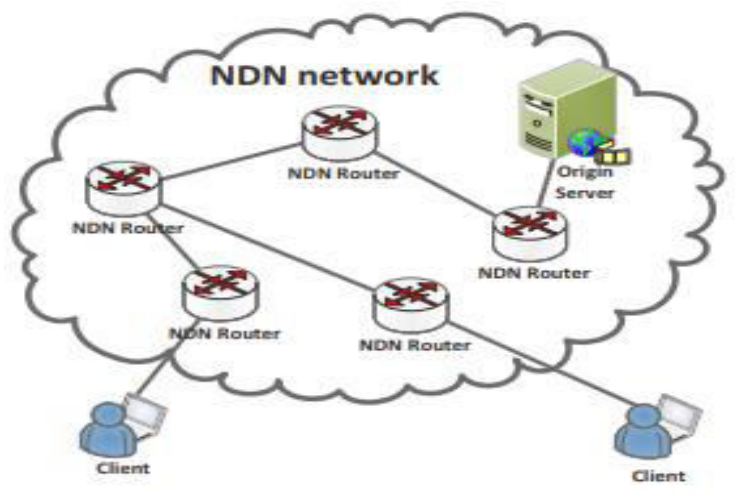

Fig. 11. System for Dynamic Adaptive Streaming over NDN (DASH over NDN) [15].

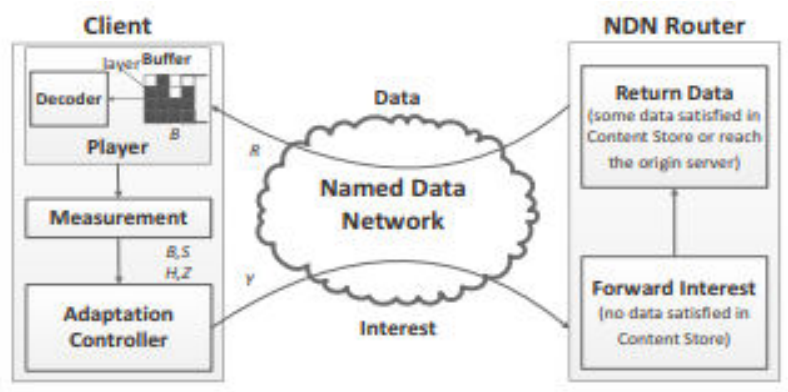

Fig. 12. The control architecture [15].

The fast and huge increase of Internet traffic motivates the development of new communication methods that can deal with the growing volume of data traffic. To this aim, named data networking (NDN) has been proposed as a future Internet architecture that enables ubiquitous in- network caching and naturally supports multipath data delivery. Particular attention has been given to using dynamic adaptive streaming over HTTP to enable video streaming in NDN as in both schemes data transmission is triggered and controlled by the clients. However, state-ofthe-art works do not consider the multipath capabilities of NDN and the potential improvements that multipath communication brings, such as increased throughput and reliability, which are fundamental for video streaming systems. Authors present a novel architecture for dynamic adaptive streaming over network coding enabled NDN [11]. In comparison to previous works proposing dynamic adaptive streaming over NDN, our architecture exploits network coding to efficiently use the multiple paths connecting the clients to the sources (see Figure 13). Moreover, this architecture enables efficient multisource video streaming and improves resiliency to Data packet losses. The experimental evaluation shows that the proposed architecture leads to reduced data traffic load on the sources, increased cache-hit rate at the in-network caches and faster adaptation of the requested video quality by the clients. The performance gains are verified through simulations in a Netflix-like scenario.

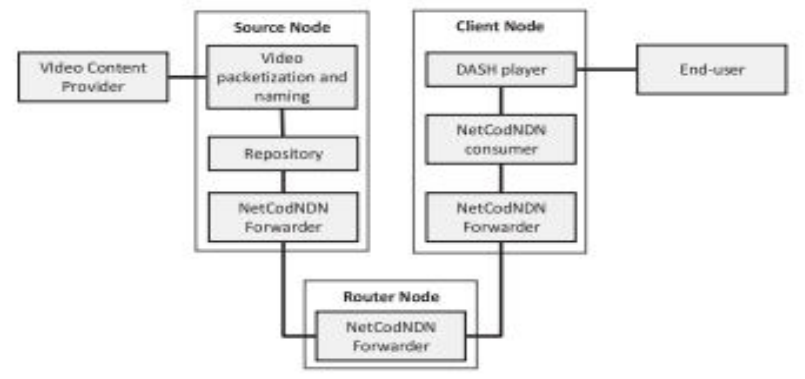

Fig. 13. Proposed architecture for adaptive video streaming over network coding enabled NDN [11].

\section{Vehicular Networks}

Information-centric networking (ICN), as a novel network paradigm, is expected to natively support mobility, multicast, and multihoming in vehicular ad hoc networks (VANETs). In this paper, the adoption of ICN principles for multimedia streaming in multihomed VANETs is investigated, with a major emphasis on the tradeoff between the quality of experience and energy efficiency (EnE). To formalize this problem, a cost optimization model is first proposed, based on queueing theory arguments. Then, a novel green information-centric multimedia streaming (GrIMS) framework is designed to drive the system toward optimal working points in practical settings. GrIMS consists of three enhanced mechanisms for on-demand cloud-based processing, adaptive multipath transmission, and cooperative innetwork caching (see Figure 14). Finally, a massive simulation campaign has been carried out, demonstrating that, thanks to its core components, the GrIMS enables flexible multimedia service provisioning and achieves an improved performance in terms of start-up delay, playbacks continuity, and EnE with respect to state-of-theart solutions. 


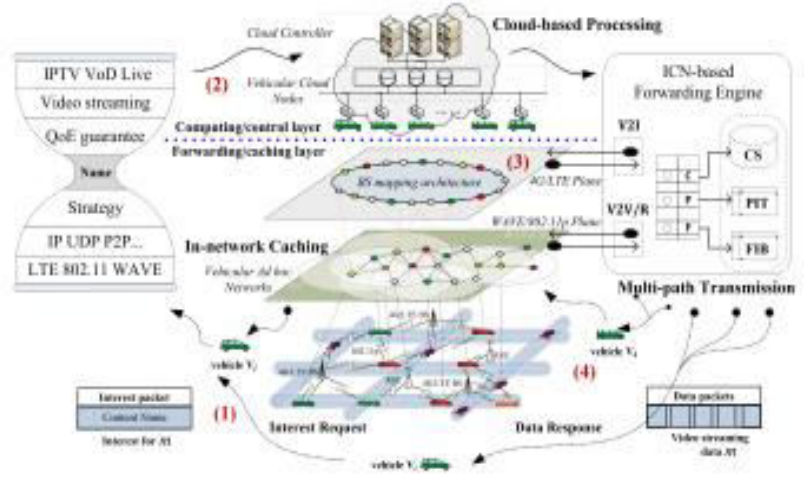

Fig. 14. GrIMS architecture [17].

\section{E. $5 G$ Systems}

The demand for rich multimedia services over mobile networks has been soaring at a tremendous pace over recent years. However, due to the centralized architecture of current cellular networks, the wireless link capacity as well as the bandwidth of the radio access networks and the backhaul network cannot practically cope with the explosive growth in mobile traffic. Recently, authors have observed the emergence of promising mobile content caching and delivery techniques, by which popular contents are cached in the intermediate servers (or middleboxes, gateways, or routers) so that demands from users for the same content can be accommodated easily without duplicate transmissions from remote servers; hence, redundant traffic can be significantly eliminated. Authors first study techniques related to caching in current mobile networks and discuss potential techniques for caching in 5G mobile networks, including evolved packet core network caching and radio access network caching (see Figure 15). A novel edge caching scheme based on the concept of content-centric networking or informationcentric networking is proposed. Using trace-driven simulations, authors evaluate the performance of the proposed scheme and validate the various advantages of the utilization of caching content in $5 \mathrm{G}$ mobile networks.

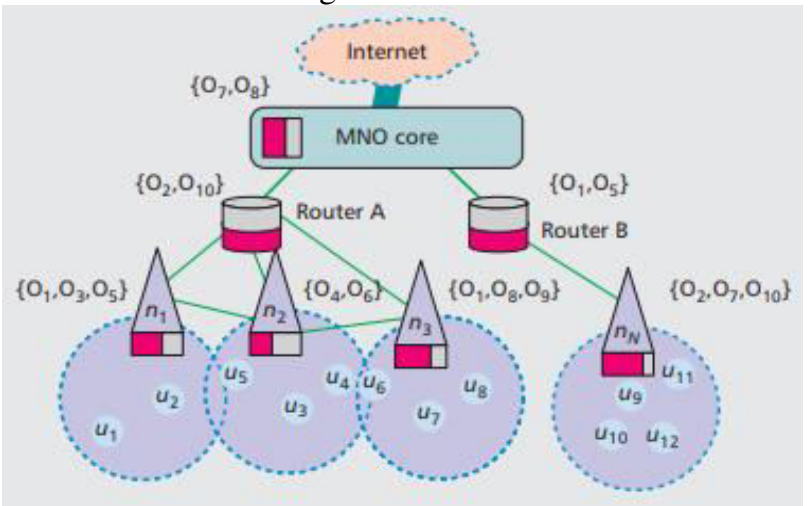

Fig. 15. An example of a cooperative caching policy [14].

\section{F. Internet of Things (IoTs)}

In view of evolving the Internet infrastructure, ICN is promoting a communication model that is fundamentally different from the traditional IP address-centric model [1].
The ICN approach consists of the retrieval of content by (unique) names, regardless of origin server location (i.e., IP address), application, and distribution channel, thus enabling in-network caching/replication and content-based security. The expected benefits in terms of improved data dissemination efficiency and robustness in challenging communication scenarios indicate the high potential of ICN as an innovative networking paradigm in the IoT domain. IoT is a challenging environment, mainly due to the high number of heterogeneous and potentially constrained networked devices, and unique and heavy traffic patterns (see Figure 16). The application of ICN principles in such a context opens new opportunities, while requiring careful design choices.

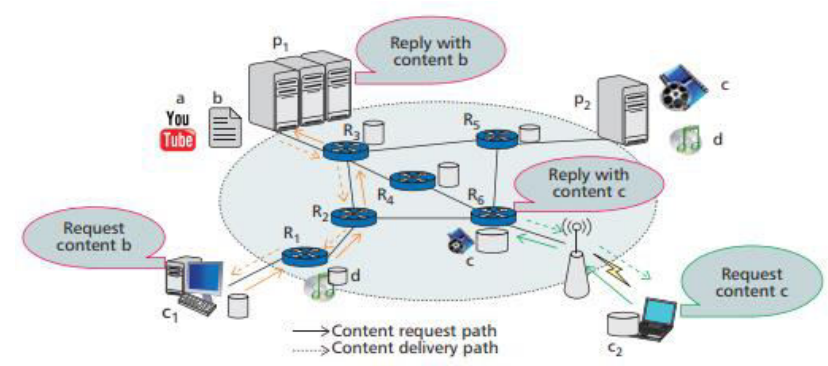

Fig. 16. Content exchange in ICN: consumers request contents that can come from any source holding a copy of them (either the original provider or a caching node) [1].

\section{G.Security}

Authors explore the synergies between a Future Internet network-layer architecture based on Information Centric Networking (ICN), and the usage of Light Field Imaging. Central to this idea is the creation of a symbiotic relationship between content names as the base network information exchange mechanism, and advanced content analysis from the rich image representations obtained by Light Field Imaging. The work done by authors increments the base ICN behavior of allowing endpoints to remotely request content to the network using only its name, and adds the capability of requesting specific aspects inside the content itself (e.g., a person's face in an image). Authors present the main functionalities and requirements associated to a solution combining ICN and Light Field Imaging for addressing video-surveillance scenarios are presented, leading to the conceptualization of the SeLF-ICN (Selectively Accessing Light Field Faces over Information Centric Networking) framework [10]. A first prototype of the framework was developed and deployed allowing the evaluation of the concepts, with results showcasing the feasibility of the proposed solution (see Figure 17). 


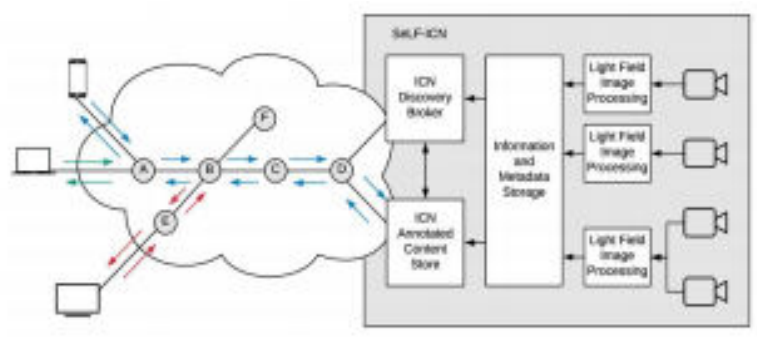

Fig. 17. SeLF-ICN architectural overview [10].

\section{CONCLUSION}

ICN has received a lot of consideration in recent years, and is a likely approach for the Future Internet design. As multimedia is the dominating traffic, it is vital to study this type of data transmission in the context of ICN. In particular, the adaptive streaming of multimedia content is a promising approach for treatment within ICN. The client has full control over the streaming session. This enables the possibility of adapting the multimedia stream to its context (e.g. network conditions, device capabilities, userQoE (Quality of Service)). These objectives are wellmatched with the standards accepted by ICN. In this article we investigate dynamic adaptive video streaming over HTTP (DASH) over networks adopting the ICN approach. In particular, we focused on seven main applications: (1) Mobile, (2) SVC-based (Scalable Video Coding), (3) Named Data Networking (NDN), (4) Vehicular Networks, (5) 5G systems, (6) Internet of Things (IoTs), and (7) Security.

\section{REFERENCES}

[1] Amadeo, Marica, Claudia Campolo, Jose Quevedo, Daniel Corujo, Antonella Molinaro, Antonio Iera, Rui L. Aguiar, and Athanasios V. Vasilakos. "Information-centric networking for the internet of things: challenges and opportunities." IEEE Network 30, no. 2 (2016): 92-100.

[2] BOUSSAHA, Ryma, Yacine Challal, Abdelmadjid Bouabdallah, Djelloul Ighit, and Lyes Tairi. "Peer-topeer collaborative video-on-demand streaming over mobile content centric networking." In 2018 IEEE 32nd International Conference on Advanced Information Networking and Applications (AINA), pp. 1053-1059. IEEE, 2018.

[3] Detti, A., Daniel Posch, Daniel Corujo, Marie-Jose Montpetit, J. Wang, Christian Timmerer, A. Azgin et al. "Adaptive Video Streaming over InformationCentric Networking (ICN)." (2016).

[4] Detti, Andrea, Bruno Ricci, and Nicola BlefariMelazzi. "Mobile peer-to-peer video streaming over information-centric networks." Computer Networks 81 (2015): 272-288.

[5] Firdhous, Mohamed Fazil Mohamed. "InformationCentric Networking." In Encyclopedia of Information
Science and Technology, Fourth Edition, pp. 65566565. IGI Global, 2018.

[6] Kurose, Jim. "Information-centric networking: The evolution from circuits to packets to content." Computer Networks 66 (2014): 112-120.

[7] Malabocchia, Fabio, Romeo Corgiolu, Maurizio Martina, Andrea Detti, Bruno Ricci, and Nicola Blefari-Melazzi. "Using information centric networking for mobile devices cooperation at the network edge." In Vehicular Technology Conference (VTC Spring), 2015 IEEE 81st, pp. 1-6. IEEE, 2015.

[8] Mpeg, I. "Information technology-dynamic adaptive streaming over http (dash)-part 1: Media presentation description and segment formats." ISO/IEC MPEG, Tech. Rep (2012).

[9] Petrangeli, Stefano, Niels Bouten, Maxim Claeys, and Filip De Turck. "Towards svc-based adaptive streaming in information centric networks." In Multimedia \& Expo Workshops (ICMEW), 2015 IEEE International Conference on, pp. 1-6. IEEE, 2015.

[10] Quevedo, Jose, Carlos Guimares, Rui Ferreira, Alireza Sepas-Moghaddam, Luis Malhadas, Rui L. Aguiar, Paulo L. Correia, and Daniel Corujo. "Selectively Accessing Light Field Face Images over Information Centric Networking." In New Technologies, Mobility and Security (NTMS), 2018 9th IFIP International Conference on, pp. 1-5. IEEE, 2018.

[11] Saltarin, Jonnahtan, Eirina Bourtsoulatze, Nikolaos Thomos, and Torsten Braun. "Adaptive Video Streaming With Network Coding Enabled Named Data Networking." IEEE transactions on multimedia 19, no. 10 (2017): 2182-2196.

[12] Samain, Jacques, Jordan Augé, Giovanna Carofiglio, Luca Muscariello, Michele Papalini, and Mauro Sardara. "Enhancing Mobile Video Delivery over an Heterogeneous Network Access with InformationCentric Networking." In Proceedings of the SIGCOMM Posters and Demos, pp. 22-24. ACM, 2017.

[13] van der Hooft, Jeroen, Stefano Petrangeli, Maxim Claeys, Jeroen Famaey, and Filip De Turck. "A learning-based algorithm for improved bandwidthawareness of adaptive streaming clients." In Integrated Network Management (IM), 2015 IFIP/IEEE International Symposium on, pp. 131-138. IEEE, 2015.

[14] Wang, Xiaofei, Min Chen, Tarik Taleb, Adlen Ksentini, and Victor Leung. "Cache in the air: exploiting content caching and delivery techniques for 
5G systems." IEEE Communications Magazine 52, no. 2 (2014): 131-139.

[15] Wu, Xiangyang, Xiaobin Tan, Yunfeng Shao, Jiawei $\mathrm{Ni}$, and Jin Zhu. "A stochastic optimization approach for dynamic adaptive scalable video streaming over NDN." In Control Conference (CCC), 2017 36th Chinese, pp. 2326-2331. IEEE, 2017.

[16] Xu, Changqiao, Wei Quan, Athanasios V. Vasilakos, Hongke Zhang, and Gabriel-Miro Muntean. "Information-centric cost-efficient optimization for multimedia content delivery in mobile vehicular networks." Computer Communications 99 (2017): 93 106.

[17] Xu, Changqiao, Wei Quan, Hongke Zhang, and Luigi Alfredo Grieco. "GrIMS: Green information-centric multimedia streaming framework in vehicular ad hoc networks." IEEE Transactions on Circuits and Systems for Video Technology (2016).

[18]Zhang, Lixia, Alexander Afanasyev, Jeffrey Burke, Van Jacobson, Patrick Crowley, Christos Papadopoulos, Lan Wang, and Beichuan Zhang. "Named data networking." ACM SIGCOMM Computer Communication Review 44, no. 3 (2014): 66-73.

\section{AUTHOR DETAILS}

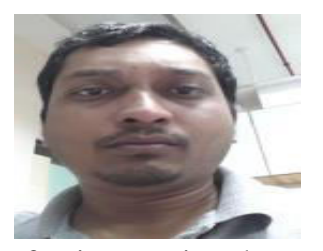

Koffka Khan received the M.Sc., and M.Phil. degrees from the University of the West Indies. He is currently a $\mathrm{PhD}$ student and has up-to-date, published numerous papers in journals \& proceedings of international repute. His research areas are computational intelligence, routing protocols, wireless communications, information security and adaptive streaming controllers.

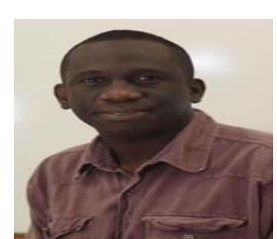

Wayne Goodridge is a Lecturer in the Department of Computing and Information Technology, The University of the West Indies, St. Augustine. $\mathrm{He}$ did is $\mathrm{PhD}$ at Dalhousie University and his research interest includes computer communications and security. 